\title{
Staphylococcus carnosus
}

National Cancer Institute

\section{Source}

National Cancer Institute. Staphylococcus carnosus. NCI Thesaurus. Code C86754.

A species of facultatively anaerobic, Gram positive, cocci shaped bacteria in the phylum Firmicutes. This species is positive for catalase and alkaline phosphatase and negative for coagulase, urease, oxidase and caseinase. It can ferment glucose, fructose, mannose, lactose, trehalose, and mannitol but not arabinose, cellobiose, maltose, sucrose, turanose or glycerol. S. carnosus is used in sausage production and is not known to be a human pathogen. 